\title{
Diphtheria Toxoid/Tetanus Toxoid/Acellular Pertussis Adsorbed, Recombinant Hepatitis B/Inactivated Poliovirus Vaccine Combined
}

National Cancer Institute

\section{Source}

National Cancer Institute. Diphtheria Toxoid/Tetanus Toxoid/Acellular Pertussis

Adsorbed, Recombinant Hepatitis B/Inactivated Poliovirus Vaccine Combined. NCI

Thesaurus. Code C91716.

A vaccine consisting of detoxified tetanus toxoid, detoxified diphtheria toxoid, acellular pertussis antigens, inactivated poliovirus (IPV) types 1,2 and 3 and hepatitis B (HBV) surface antigen, with active immunizing activities against diphtheria, tetanus, pertussis, hepatitis $\mathrm{B}$, and poliomyelitis. The acellular pertussis components in this vaccine, produced by Bordetella pertussis, are detoxified pertussis toxin (PT), filamentous hemagg lutinin (FHA) and pertactin (PRN). Upon intramuscular injection, this vaccine activates the immune system to develop antibodies against tetanus toxin, diphtheria toxin, B. pertussis antigens, polioviruses and HBV. The diphtheria and tetanus toxoids and pertussis antigens (inactivated PT, FHA, and pertactin) are adsorbed onto aluminum hydroxide; the hepatitis B antigen is adsorbed onto aluminum phosphate. 